\title{
Real-time profile of microcantilevers for sensing applications
}

\author{
J. Mertens, M. Álvarez, and J. Tamayo ${ }^{\text {a) }}$ \\ BioNanoMechanics Lab, National Centre for Microelectronics, IMM-CNM, CSIC Isaac Newton 8 (PTM), \\ Tres Cantos 28760, Madrid, Spain
}

(Received 19 July 2005; accepted 3 October 2005; published online 28 November 2005)

\begin{abstract}
An optical readout technique has been developed for real-time monitoring of the profile of microcantilever arrays for sensing applications. The technique is based on the automated two-dimensional scanning of a laser beam by using voice-coil actuators. Cantilever profiles are obtained with subnanometer resolution and a processing speed of about ten cantilevers per second. The technique is applied for real-time monitoring of the adsorption of the alkylthiol mercaptohexanol in an aqueous environment by using an array of five microcantilevers. Molecular adsorption produces a cantilever strain that significantly differs from the Stoney's model. Main strain changes are strongly located near the cantilever clamping. (C) 2005 American Institute of Physics. [DOI: 10.1063/1.2136410]
\end{abstract}

Nanomechanical sensors are based on the measurement of the strain induced on suspended microstructures such as cantilevers by molecular adsorption. ${ }^{1-9}$ A crucial element of these devices is the readout technique that ideally should satisfy (i) high sensitivity for measuring the cantilever strain as well as (ii) the capability for readout of microcantilever arrays for simultaneous detection of different targeted molecules and also for isolation of molecular recognition signals from other nonspecific signals. Optical techniques based on beam deflection and interferometry have been used for measuring the relative displacement of a single point near the cantilever free end with picometer resolution. ${ }^{10}$ However, simplistic models must be assumed to deduce the strain of the cantilever from the displacement measurement. Recently, two research groups have overcome this limitation by developing optical techniques for measuring the cantilever profile based on an array of eight laser emitters and phase shifting interferometry, respectively. ${ }^{11,12}$ On the other hand, the implementation of optical techniques for readout of microcantilever arrays implies important restrictions in the cantilever geometry, cantilever interdistance, and the number of cantilevers. $^{13,14}$

Here we present a technique for optical readout of the profile of microcantilever arrays with subnanometer resolution and a processing speed of about ten cantilevers per second.

The readout technique combines the optical beam deflection method and the automated two-dimensional scanning of a single laser beam by voice-coil actuators. Figure 1(a) shows a schematic drawing of the experimental setup. A $3 \mathrm{~mW}$ laser diode is mounted on two perpendicular linear voice coil actuators that allow two-dimensional nonhysteretic displacement over a range of several millimeters at speeds of up to $50 \mathrm{~mm} / \mathrm{s}$ with an accuracy of $100 \mathrm{~nm}$. One of the scanning axes is oriented parallel to the cantilever longitudinal axis. The laser beam is directed to illuminate the cantilever array and a two-dimensional linear position detector (PSD) is arranged to collect the reflected beams. A convergent lens is used to decrease the spot size of the laser on the cantilevers to 5-10 $\mu \mathrm{m}$, approximately. Two scan trajec-

\footnotetext{
a) Author to whom correspondence should be addressed; electronic mail:
} jtamayo@imm.cnm.csic.es tories are executed in order to obtain the cantilever profile in the array [Fig. 1(b)]. In a first scan, the laser beam is displaced perpendicularly to the longitudinal cantilever axis for identification of the cantilever positions by detecting maxima of light intensity in the PSD. ${ }^{15}$ Second scans parallel to the longitudinal cantilever axis are, then, performed from the reference positions identified in the first scan, in order to measure the cantilever profiles.

A change of the local slope at the cantilever surface results into a displacement of the reflected laser spot on the PSD, referred to as $s$. Thus, the displacement recorded by the PSD during the second scans are described by

$$
s(x) \cong 2 D \frac{d z}{d x}(x)+x \cos \beta,
$$

where $z(x)$ is the cantilever profile along its longitudinal axis, $D$ is the distance between the cantilever and PSD, and $\beta$ is the angle between the incident laser beam and the cantilever normal at its rest position. Second summand accounts for the effect of the laser beam displacement, which is generally small compared to the first summand associated to the slope variations. The cantilever profiles are obtained by integrating Eq. (1),

$$
z(x)=\frac{1}{2 D} \int_{0}^{x} s\left(x^{\prime}\right) d x^{\prime}-\frac{1}{4} \frac{x^{2}}{D} \cos \beta .
$$

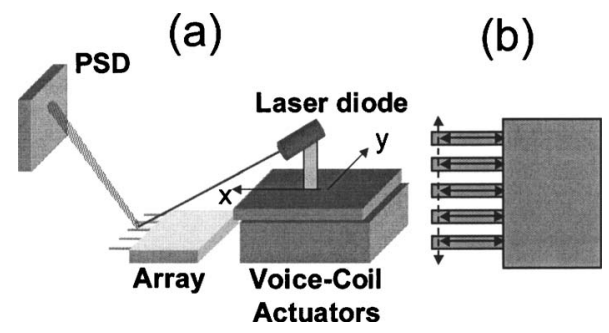

FIG. 1. Schematic depiction of the readout technique for real-time profiling of cantilever arrays. (a) Depiction of the experimental setup. (b) Schematic of the scan trajectories performed over the cantilever array. The dotted line represents the first scan trajectory performed to detect the position of the cantilevers. Continuous lines represent the second scan along each cantilever. 

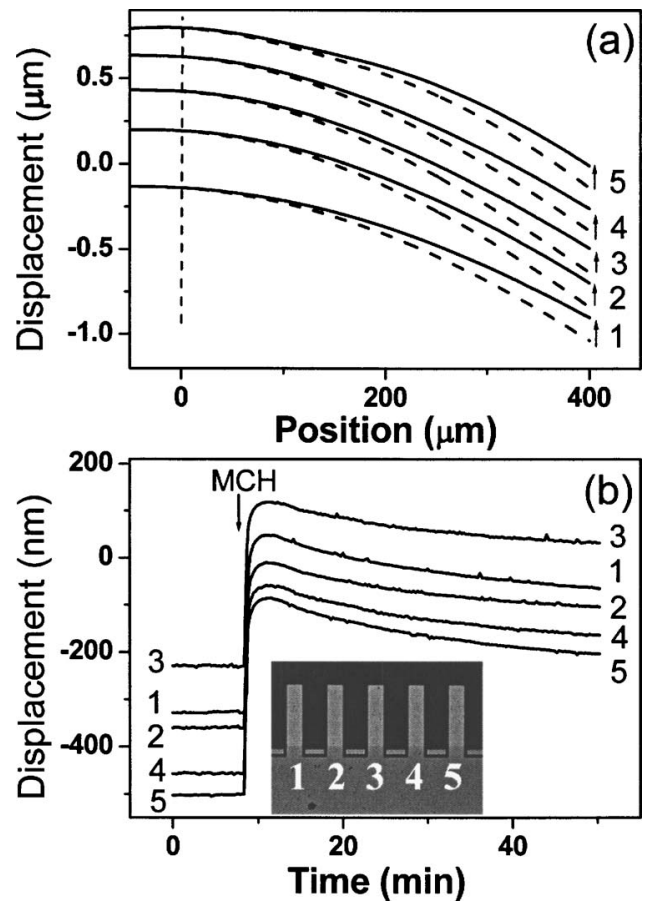

FIG. 2. (a) Displacement profile of five gold-coated silicon cantilevers of an array before (dotted lines) and after adsorption (solid lines) of $\mathrm{MCH}$. The vertical dashed line indicates the clamping position. (b) Real-time measurement of the displacement of the free end of the five microcantilevers. The arrow indicates the moment when the MCH begins to adsorb on the cantilevers. The data is obtained from the continuous measurement of the cantilever profiles such as those shown in (a). The inset in (b) shows an optical image of the cantilever array used in the experiments. The measurements were performed in water and the cantilevers length, width, and thickness were 400,100 , and $1 \mu \mathrm{m}$, respectively. The array pitch distance was $250 \mu \mathrm{m}$.

The technique was applied for measuring the temporal evolution of the mechanical response of a silicon array composed by five microcantilevers coated with a thin gold layer on one side, during the exposure to the alkylthiol mercaptohexanol $(\mathrm{MCH})$ in an aqueous solution. $\mathrm{MCH}$ forms highly packed monolayers on the gold-coated side of cantilevers due to the strong bond between the thiol end and the gold.

Figure 2(a) shows the resulting cantilever profiles measured in water before and after exposure of the cantilevers to $\mathrm{MCH}$. The scan speed was set to $500 \mu \mathrm{m} / \mathrm{s}$ and the longitudinal axis of the cantilever was selected with a precision of $100 \mathrm{~nm}$. We did not observe significant variations in the cantilever profile for small offsets with respect to the centre axis. The cantilever free ends were determined at the positions where the total light intensity collected by the PSD falls to the half. The cantilevers displace upward (toward the silicon side) 360-410 nm. The displacement arises from the compressive surface stress on the gold due to the strong attachment of the $\mathrm{MCH}$ molecules, which expands the gold surface (bottom) with respect to the silicon side (top). From the profile data, the displacement of the cantilever free end is obtained and monitored in real time to follow the adsorption process [Fig. 2(b)]. The cantilevers significantly displace upward during the first seconds of exposure to $\mathrm{MCH}$ indicating that a highly packed monolayer is quickly formed. Then the cantilevers slowly displace downward during several minutes. This is attributed to the detachment of loosely bound molecules as well as long-term reorganization processes in the monolayer. tion, which can be described as an angular translation of the
the monolayer.
Downloaded 05 Nov 2009 to 161.111.235.169. Redistribution subject to AlP license or copyright; see http://apl.aip.org/apl/copyright.jsp

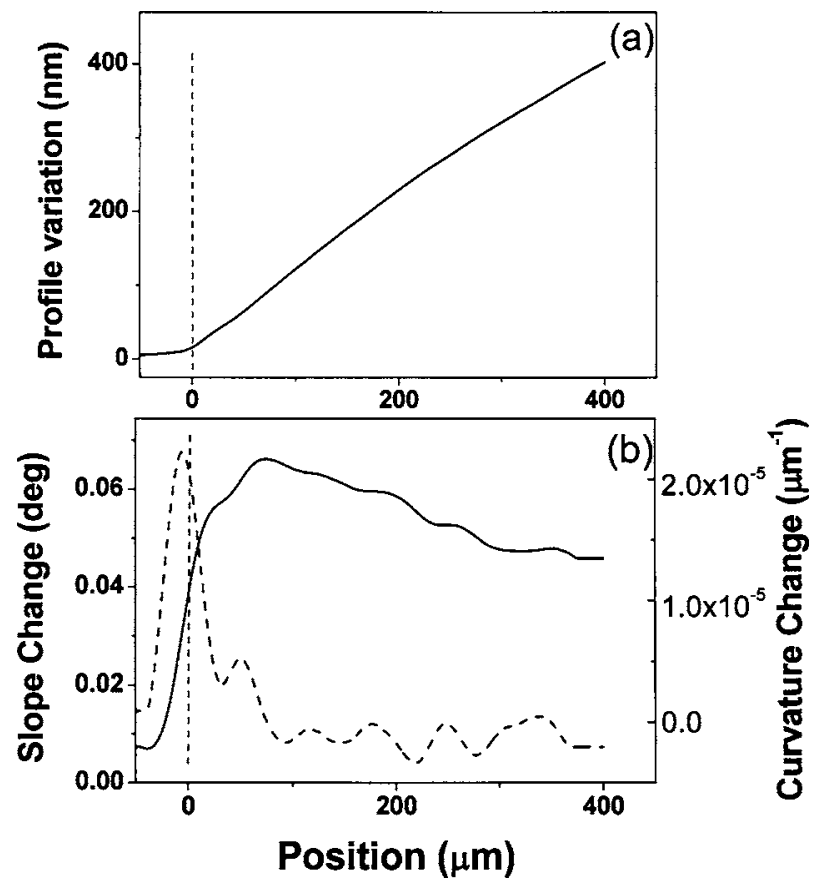

FIG. 3. (a) Change of the displacement profile due to $\mathrm{MCH}$ adsorption in water for one of the cantilevers of the array shown in the inset of Fig. 2. (b) Profiles of the change of the slope (solid line) and curvature (dotted line) due to $\mathrm{MCH}$ adsorption in water. The vertical dashed arrow indicates the clamping position.

To elucidate the strain mechanisms of the cantilevers during molecular adsorption, the initial cantilever profiles were subtracted from the profiles after $\mathrm{MCH}$ adsorption. Figure 3(a) shows the profile variation corresponding to one of the cantilevers for major simplicity, although the results were found reproducible for all the cantilevers of the array. A maximum displacement of $408 \mathrm{~nm}$ is found at the free end of the cantilever. Notice that this technique provides the real deflection. In the usual optical beam deflection technique, the deflection value is obtained by assuming a cantilever bending with uniform curvature due to the differential surface stress between opposite sides, a situation that is not real, as shown below. From the differential displacement profile, the tilt angle variation and curvature variation profiles are calculated, as shown in Fig. 3(b). The effect of the compressive surface stress onto the gold surface induces a strong curvature change at the clamping region of about 2.2 $\times 10^{-5} \mu \mathrm{m}^{-1}$, that quickly decays toward the free cantilever end. In fact, the mean curvature change in the free region of the cantilever is much smaller and with opposite sign, of about $1.33 \times 10^{-6} \mu \mathrm{m}^{-1}$. In a similar way, a noticeable slope variation is found near the clamping region that continuously decreases along the cantilever length. The slope variation at the cantilever end is of about 25\%-30\% smaller than near the clamping.

The effect of differential surface stress on the cantilever strain is usually described by applying the Stoney's model discovered almost one century ago. This model was originally applied to understand the bending of plates under the deposition of metallic films by electrolysis. The model accurately predicts a uniform curvature radius inversely proportional to the tension (surface stress) of the deposited film. Stoney's model clearly deviates from the picture here obtained for the cantilever strain induced by molecular adsorp- 
beam due to the curvature change in the region of the clamp. ${ }^{16}$ We attribute this different behavior to that Stoney's equation applies for plates that are unrestrained along their edges, whereas cantilevers are rigidly clamped at one of the ends. ${ }^{17-19}$ However, a major theoretical modelling is required in order to establish the basis on the mechanical response of cantilevers induced by molecular adsorption.

In conclusion, an optical technique has been developed for real-time profiling of cantilever arrays for biological and chemical sensing. The technique has been applied for measuring the profile change of a cantilever array during the molecular adsorption of $\mathrm{MCH}$ in a water solution. Adsorption-induced strain significantly deviates from the expected behavior (Stoney's model). The implications of the results are of crucial relevance for the understanding of the bending mechanisms in cantilever sensors as well as for optimization of readout techniques.

The authors would like to acknowledge A. Calle, L. Lechuga, and M. Calleja for their helpful suggestions. This work was supported by Spanish Ministry of Science (GEN2001-4856-C13-11).

${ }^{1}$ N. Lavrik, M. Sepaniak, and P. Datskos, Rev. Sci. Instrum. 75, 2229 (2004).

${ }^{2}$ R. Raiteri, M. Grattarola M., H. J. Butt, and P. Skládal, Sens. Actuators B 79, 115 (2001).

${ }^{3}$ L. A. Pinnaduwage, A. Gehl, D. L. Hedden, G. Muralidharan, T. Thundat,
R. T. Lareau, T. Sulchek, L. Manning, B. Rogers, M. Jones, and J. D. Adams, Nature 425, 474 (2003).

${ }^{4}$ R. McKendry, J. Zhang, Y. Arntz, T. Strunz, M. Hegner, H. P. Lang, M. K. Baller, U. Certa, E. Meyer, H.-J. Güntherodt and Ch. Gerber, Proc. Natl. Acad. Sci. U.S.A. 99, 9783 (2002).

${ }^{5}$ L. B. Sharos, A. Raman, S. Crittenden, and R. Reifenberger, Appl. Phys. Lett. 84, 4638 (2004).

${ }^{6}$ G. Wu, R. H. Datar, K. M. Hansen, T. Thundat, R. J. Cote, and A. Majumdar, Nat. Biotechnol. 19, 856 (2001).

${ }^{7}$ M. Álvarez, L. G. Carracosa, M. Moreno, A. Calle, A. Zaballos, L. M. Lechuga, C. Martínez-A, and J. Tamayo, Langmuir 20, 9663 (2004).

${ }^{8}$ B. Ilic, Y. Yang, and H. G. Craighead, Appl. Phys. Lett. 85, 2604 (2004).

${ }^{9}$ C. A. Savran, T. P. Burg, J. Fritz, and S. R. Manalis, Appl. Phys. Lett. 83, 1659 (2003).

${ }^{10}$ C. A. J. Putman, B. G. De Grooth, N. F. Van Hulst, and J. Greve, J. Appl. Phys. 72, 6 (1992).

${ }^{11}$ S. Jeon and T. Thundat, Appl. Phys. Lett. 85, 1083 (2004).

${ }^{12}$ M. Helm, J. J. Servant, F. Saurenbach, and R. Berger, Appl. Phys. Lett. 87, 064101 (2005).

${ }^{13}$ H. P. Lang, R. Berger, C. Andreoli, J. Brugger, M. Despont, P. Vettiger, Ch. Gerber, J. K. Gimzewski, J. P. Ramseyer, E. Meyer, and H.-J. Güntherodt, Appl. Phys. Lett. 72, 383 (1998).

${ }^{14}$ T. Sulchek, R. J. Grow, G. G. Yaralioglu, S. C. Minne, C. F. Quate, S. R. Manalis, A. Kiraz, A. Aydine, and A. Atalar, Appl. Phys. Lett. 78, 1787 (2001).

${ }^{15}$ M. Alvarez and J. Tamayo, Sens. Actuators B 106, 687 (2005).

${ }^{16}$ P. A. Rasmussen, O. Hansen, and A. Boisen, Appl. Phys. Lett. 86, 2035 (2005).

${ }^{17}$ N. Guyot, Y. Harmand and A. Mézin, Int. J. Solids Struct. 41, 5143 (2004).

${ }^{18}$ J. E. Sader, J. Appl. Phys. 89, 2911 (2001).

${ }^{19}$ Y. Zhang, Q. Ren, and Y. Zhao, J. Phys. D 37, 2140 (2004). 